\title{
Parasitosis y síndrome de intestino irritable
}

\author{
Catalina Ibarra, Valentina Herrera, Edith Pérez de Arce, Luis Carlos Gil, \\ Ana María Madrid, Lucía Valenzuela y Caroll J. Beltrán
}

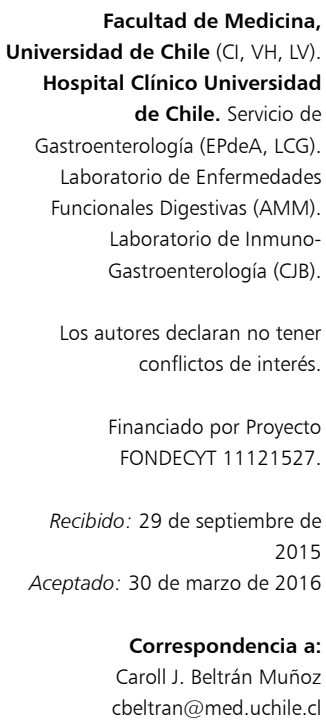

Facultad de Medicina Universidad de Chile $(\mathrm{Cl}, \mathrm{VH}$, LV) Hospital Clínico Universidad de Chile. Servicio de Gastroenterología (EPdeA, LCG) Laboratorio de Enfermedades Funcionales Digestivas (AMM)

Laboratorio de InmunoGastroenterología (CJB)

Los autores declaran no tener conflictos de interés.

Financiado por Proyecto FONDECYT 11121527

Recibido: 29 de septiembre de

Aceptado: 30 de marzo de 2016

Correspondencia a: Caroll J. Beltrán Muñoz cbeltran@med.uchile.cl

\section{Parasitosis and irritable bowel syndrome}

Irritable bowel syndrome (IBS) is a functional disorder of the gastrointestinal tract characterised by multifactorial aetiology. In IBS physiopathology are involved diverse factors between them biological, psychosocial, and environmental components which affect the immune activation status of gut mucosa. Among these factors is recognized the intestinal parasitosis. Post-infection IBS (PI-IBS) is recognised as a subgroup of functional disorders whose symptoms onset appear after a symptomatic intestinal infection caused by microbial agents. There are few studies regarding of relationship between IBS and intestinal parasitosis in Chile. However, is has been well described a positive association between IBS and Blastocystis hominis infections, one of prevalent parasites in Chile. In other countries, is also described a relationship between IBS and amebiasis and giardiasis. Both, characterized by a common mode of transmission through water as well as contaminated food. Because the high prevalence of parasitosis in our country it is necessary to expand the association studies to clarify the strength of the parasites ethiology in IBS.

Key words: Irritable bowel syndrome, Post-infection irritable bowel syndrome, Intestinal parasitosis, Blastocystis hominis, Entamoeba histolytica, Giardia intestinalis.

Palabras clave: Síndrome de intestino irritable, síndrome de intestino irritable post-infeccioso, parasitosis intestinal, Blastocystis hominis, Entamoeba histolytica, Giardia intestinalis.

\section{Introducción}

$\mathrm{E}$ 1 síndrome de intestino irritable (SII) es uno de los trastornos digestivos funcionales más frecuentes, que afecta entre 10 y $25 \%$ de la población mundial $^{1}$. Si bien los reportes de prevalencia en Chile son escasos, en un estudio realizado a una población no seleccionada de personas asistentes a centros comerciales de la Región Metropolitana, indicó que alrededor de 28,6\% de los individuos estudiados presentaron sintomatología compatible con SII bajo criterios Roma II, principalmente para el género femenino, siendo esta frecuencia levemente mayor a lo reportado en el resto de Latinoamérica ${ }^{2}$. Si bien no causa mortalidad, este cuadro provoca gran deterioro en la calidad de vida de los pacientes ${ }^{3}$, ausentismo laboral y aumento en los gastos en salud pública ${ }^{4}$.

Pese a que en su etiología no está claramente definida la presencia de una alteración orgánica específica, se le considera como un trastorno multifactorial debido a que factores genéticos, ambientales y psicosociales, entre otros, han sido relacionados con la aparición y mantención de su sintomatología ${ }^{5}$. Entre los numerosos factores ambientales se incluye la asociación con infecciones microbianas, dentro ellas las parasitosis ${ }^{6}$.

Las parasitosis intestinales son prevalentes en países subdesarrollados con mal manejo de residuos biológicos, especialmente excretas, y un pobre sistema de tratamiento de agua potable ${ }^{7}$. En Chile, a pesar de poseer políticas de salud pública dirigidas a la prevención de enfermedades infecciosas, las parasitosis intestinales aún son frecuentes sobre todo en niños, con reportes de $13 \%$ bajo 5 años con diarrea, pesquisados en centros centinelas ${ }^{8}$ y de 55 y $76 \%$ en niños asintomáticos, pertenecientes a las comunas de Colina y Talca, respectivamente $e^{9,10}$.

La literatura científica internacional plantea la existencia de una asociación positiva entre la presencia de parásitos intestinales y la aparición de síntomas de este desorden funcional digestivo, reportes que se caracterizan por ser controversiales debido a la heterogeneidad de los grupos de individuos analizados y del área geográfica en estudio ${ }^{1}$. El objetivo de esta revisión es exponer los antecedentes actuales respecto a la relación que existe entre las parasitosis intestinales y el desarrollo de SII, con el fin de generar una breve discusión respecto a los posibles mecanismos fisiopatológicos que se encuentran asociados a esta relación.

\section{El síndrome de intestino irritable}

El SII es un trastorno digestivo funcional que se caracteriza por la presencia de malestar o dolor abdominal asociados a cambios en la defecación. Su causa orgánica aún no está bien esclarecida ${ }^{11}$ lo que lleva a que su diagnóstico sea fundamentalmente clínico basado en 
los criterios establecidos por la Fundación ROMA III ${ }^{12}$ y la exclusión de otras enfermedades con causa orgánica. Según la frecuencia y consistencia de las deposiciones el SII se clasifica en 4 subtipos: SII diarreico (SII-D), SII con estreñimiento o constipado (SII-E o SII-C), SII de subtipo mixto (SII-M) y SII indeterminado (SII-I) (Figura 1) ${ }^{13}$.

En la fisiopatología del SII participan diversos factores, entre ellos ha sido ampliamente descrita la presencia de alteraciones biológicas que afectan la comunicación neuro-inmune de la mucosa intestinal ${ }^{14}$, proceso que se ve favorecido frente a condiciones genéticas predisponentes ${ }^{15}$. Dentro de los mecanismos biológicos alterados se incluyen: la activación de células inmunes en la mucosa intestinal, tales como linfocitos $\mathrm{T}$, linfocitos B y mastocitos; las alteraciones en la composición y aumento del número de la microbiota intestinal, que llevan al procesamiento anormal de moléculas presentes en la dieta; y la mayor liberación de sustancias bioactivas por células enterocromafines $(\mathrm{EC})^{11,14,16,17}$. La respuesta inflamatoria intestinal es reforzada por una mayor actividad nerviosa entérica, relacionada al aumento de la sensibilidad visceral e incremento en la estimulación de terminales nerviosos aferentes y a la alteración del eje intestino-cerebro en estos pacientes ${ }^{18}$. Un factor patogénico relevante que contribuye al desarrollo de SII es la aparición de episodios de gastroenteritis infecciosa aguda, muchas de las cuales son causadas por parasitosis intestinales, especialmente los protozoarios ${ }^{6}$. Esto ha llevado a reconocer a un subgrupo especial de pacientes con SII, el SII post-infeccioso.

\section{Respuesta inmune en el síndrome de intestino irritable post-infeccioso}

El síndrome de intestino irritable post infeccioso (SII-PI) se define como la aparición de sintomatología concordante con los criterios de SII, inmediatamente después de una enfermedad intestinal infecciosa aguda ${ }^{19,20}$.

El primer reporte que hace referencia a este cuadro fue realizado por Chaudhary y Troulove en 1962 en relación a sujetos con disentería amebiana y la persistencia de síntomas abdominales tras superar el episodio agudo ${ }^{21}$. Si bien no existe consenso respecto a la fisiopatología del SII-PI se postula que la adquisición de una infección aguda del tracto intestinal genera un estado inflamatorio crónico sostenido que puede permanecer incluso tras la erradicación del agente infeccioso ${ }^{22}$. Como se esquematiza en la Figura 2, esta fase inflamatoria del SII se caracteriza por la presencia de un mayor grado de infiltración y elevada actividad de células inmunes, tales como leucocitos, linfocitos, mastocitos y células endocrinas, en la mucosa. El estado de inflamación permanente está asociado además a una alteración de la permeabilidad paracelular de la barrera epitelial del intestino, lo que favorece el paso de antígenos proveniente del lumen intestinal hacia la mucosa, muchos de ellos provenientes de la dieta y de la microbiota. La estimulación aumentada del sistema inmune local trae como consecuencia el aumento en la liberación de mediadores inflamatorios y el reclutamiento de mayores componentes celulares a la mucosa intestinal, perpetuando de esta manera el ciclo de inflamación crónica $^{11,23}$. Se ha demostrado que la respuesta inmune local

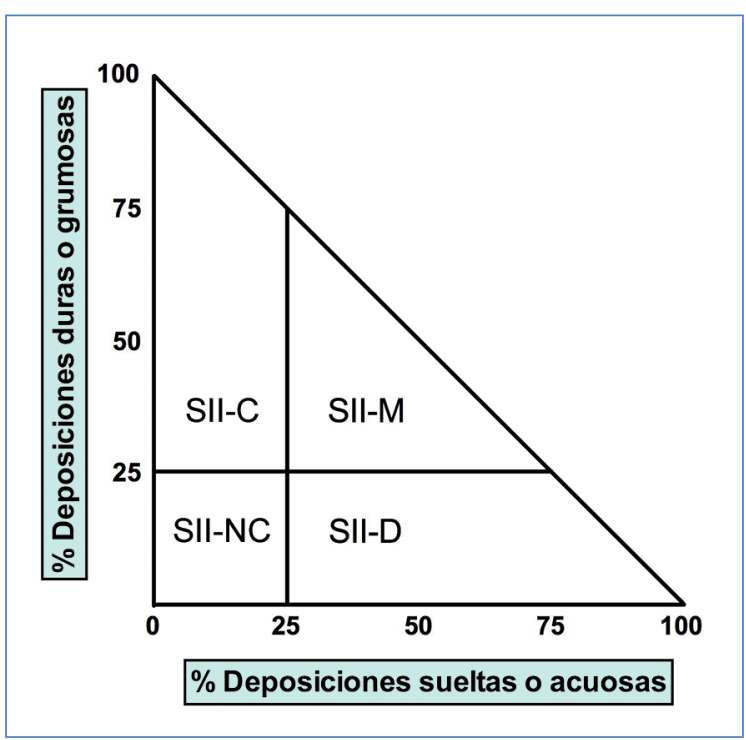

Figura 1. Esquema en dos dimensiones que representa los cuatro subtipos de síndrome de intestino irritable, basados en la forma de las deposiciones en el tiempo. SII-C: SII constipado o con estreñimiento; SII-D: SII con diarrea; SII-M: SII mixto; SII-NC: SII no clasificable (Adaptado de Longstreth GF, Thompson WG, Chey WD, et al. Functional bowel disorders. Gastroenterology 2006; 130: 1480-91).

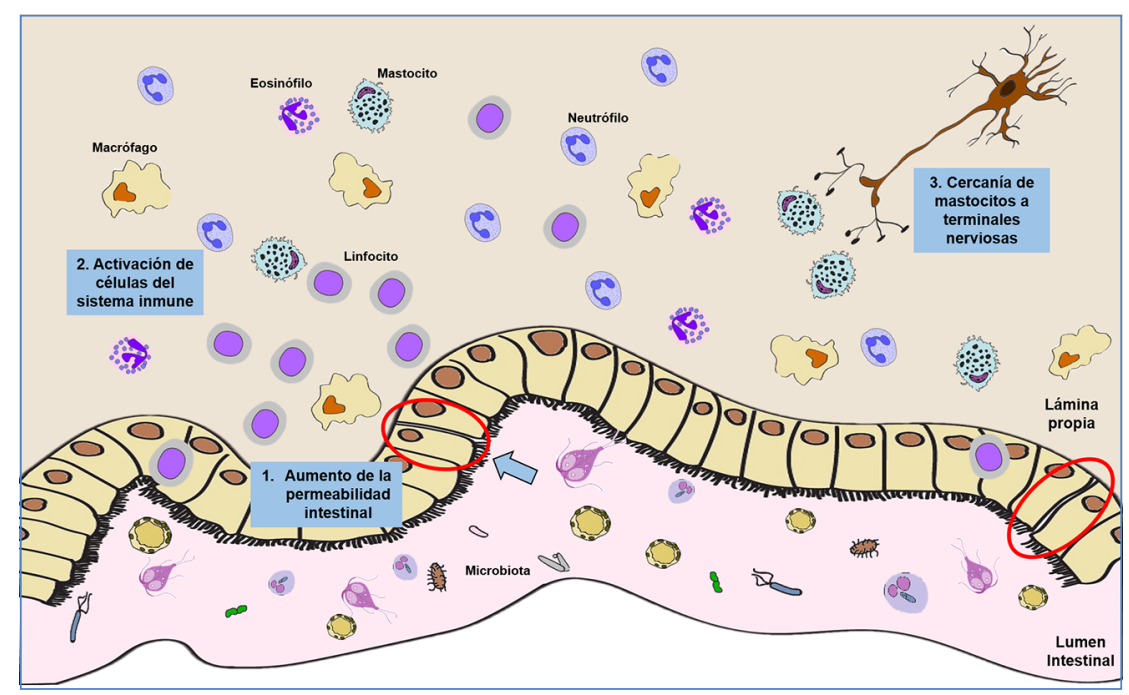

Figura 2. Ilustración que representa los mecanismos inmunes activados en la mucosa intestinal, durante el desarrollo del Síndrome Intestino Irritable Post-Infeccioso (SII-PI). Los cambios en la permeabilidad de la barrera epitelial (1) sumado a las alteraciones en la composición de la microbiota intestinal, inducen la activación de células inmune en mucosa (2) que predisponen a una inflamación de bajo grado persistente en los pacientes con SII. El aumento de mediadores inflamatorios induce el reclutamiento de células inmunes en el intestino, entre ellos mastocitos. La activación de mastocitos intestinales, trae como consecuencia la liberación de mediadores solubles que son capaces de activar las terminaciones nerviosas entéricas (3), mecanismo asociado al aumento de la sensibilidad visceral en pacientes con SII. 
activada puede ser extrapolada a nivel periférico. Es así como Ohman L. y cols., describen una aumentada activación de linfocitos $\mathrm{T}$, tanto en el colon como periférica en pacientes con $\mathrm{SII}^{24}$. La mayor expresión de citoquinas pro-inflamatorias, tanto en el plasma ${ }^{25}$ como por células mononucleares de sangre periférica estimuladas in vitro ${ }^{26}$, sugieren un estado de predisposición sistémica a la activación inmune en estos pacientes.

Un hallazgo importante es la alteración significativa, tanto en el recuento de la células EC en el colon como en las concentraciones de serotonina (5-HT) en sangre, tras infecciones entéricas bacterianas y parasitarias ${ }^{27}$. En modelos animales, se ha demostrado que además de la hiperplasia de EC, existe un desarrollo anormal en los patrones de motilidad intestinal del colon, efecto que es revertido tras la administración de dexametasona ${ }^{28}$.

Las células cebadas o mastocitos son elementos centrales en la aparición de la hipersensibilidad periférica o aumento de la percepción de dolor en los pacientes con SII, debido a que se localizan en próxima cercanía a fibras nerviosas sensoriales (Figura 2) ${ }^{29}$. Estudios recientes han demostrado que los mastocitos median la actividad inflamatoria de la mucosa intestinal en respuesta a infecciones parasitarias, tales como infección por Entamoeba histolytica. En estos casos, se ha visto un aumento de secreción de IL-8 por mastocitos intestinales en respuesta a mediadores solubles secretados por el parásito ${ }^{30}$. Además, en modelos animales de giardiasis, se ha demostrado que la captación activa de antígenos durante el pico de la infección parasitaria aumenta el reclutamiento de mastocitos en la mucosa y en el tejido conectivo intestinal, como un mecanismo de defensa natural antiparasitario ${ }^{31}$. Estos hallazgos sugieren un papel central para los mastocitos intestinales en el desarrollo de respuestas inflamatorias que favorecen la estimulación neuro-inmune en el SII-PI.

Los factores de riesgo para el desarrollo de la SII-PI son diversos. Estos incluyen, en orden de importancia: la duración de la infección, la patogenicidad del microorganismo infectante, el tabaquismo, los marcadores de la inflamación de la mucosa, el género femenino y los síntomas de depresión psicológica, entre otros ${ }^{20,23,27,32}$. Además, se ha visto que el SII es un desorden común en países donde la infestación parasitaria crónica es endémica, sugiriendo a este factor ambiental como un componente de susceptibilidad al desarrollo de SII-PI.

\section{Parasitosis y el SII en Chile}

En Chile, existe sólo un estudio dirigido a establecer la relación entre el SII con las parasitosis intestinales y corresponde al grupo de Madrid, AM. y cols., realizado en muestras de deposiciones de pacientes chilenos con SII que acudían al Servicio de Gastroenterología del Hospital Clínico Universidad de Chile. Se incluyeron 80 pacientes con SII (65 de ellos mujeres), donde en 34 (42,5\%) de los sujetos se encontró parasitosis intestinal: 32 de ellos portadores de Blastocystis hominis y dos de E. histolytica. Se estimó que los pacientes con SII presentaron un riesgo de 3,74 (Odd Ratio (OR)) de tener una parasitosis intestinal $(\mathrm{p}<0,0001)$, siendo el subtipo de SII-alternante o mixto, el que presentó una prevalencia significativamente mayor frente a los otros subtipos ${ }^{33}$. Estos resultados sugieren la existencia de una asociación entre la parasitosis intestinal y el SII en nuestro país, y plantea la necesidad de contar con investigaciones dirigidas no sólo a evaluar esta relación en un mayor número de pacientes, sino también a evaluar los posibles mecanismos fisiopatológicos involucrados en esta asociación.

A continuación se describen algunas evidencias respecto a tres de los agentes protozoarios más frecuentemente asociados al desarrollo del SII: B. hominis, E. histolytica y Giardia intestinalis (también conocida como G. lamblia o G. duodenalis).

\section{Blastocystis hominis}

Blastocystis hominis es un parásito protozoario entérico, unicelular, que durante su desarrollo presenta cuatro estadios o fases: vacuolar, granular, ameboide y quística. El quiste, resistente a condiciones ambientales adversas, es ingerido, usando como vehículo aguas y alimentos contaminados con heces, estableciéndose posteriormente en el íleon y el colon. Esta parasitosis corresponde a una zoonosis, ya que puede infectar, tanto al hombre como a otros animales, quienes actúan como reservorio ${ }^{34}$.

Estudios epidemiológicos estiman una prevalencia de B. hominis bajo $5 \%$ en países industrializados, pudiendo llegar hasta 30 a $60 \%$ en países en desarrollo ${ }^{35}$. En nuestro país, en un estudio realizado en pre-escolares y escolares de la comuna de Colina el 2003 , se reportó que $B$. hominis fue la parasitosis más frecuente, con una prevalencia global de 41,3\% ${ }^{9}$. Estudios de prevalencia realizados en Talca en población escolar y pre-escolar, reportaron cifras de portación de $B$. hominis de hasta $72,9 \%$ entre los años 2005 y $2007^{10}$.

El papel patogénico de $B$. hominis es controversial al igual que su asociación con $\mathrm{SII}^{36}$. Estudios recientes han mostrado una mayor prevalencia de $B$. hominis en pacientes con SII (31-60\%) versus controles (7-16\%) $)^{37-40}$. Entre éstos destacan los estudios realizados por Yakoob y cols., quienes en una primera aproximación detectaron $B$. hominis a partir de cultivo fecal en $32 \%$ de pacientes con SII versus $7 \%$ en controles $(\mathrm{p}<0,001)^{41}$. Años más tarde, los mismos autores reportaron, a través de un estudio realizado a un grupo de 171 pacientes con SII subtipo diarreico y 159 controles, la presencia de $B$. hominis por técnica de microscopia directa en deposiciones, cultivo fecal y reacción de polimerasa en cadena (RPC) de 49, 53 y 44\%, respectivamente, versus 24,16 y $21 \%$ en controles usando 
las mismas técnicas $(\mathrm{p}<0,001)^{42}$. Pese a estos resultados, otros estudios no logran demostrar la asociación entre SII

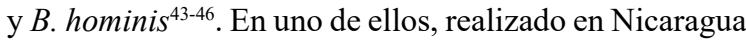
en 163 pacientes con SII y 194 controles, se demostró que sólo $8 \%$ de los casos presentó B. hominis versus $10,3 \%$ en los controles ${ }^{46}$. Las diferencias metodológicas respecto a la selección de los sujetos de estudio, técnicas de diagnóstico de Blastocystis (cultivos, estudio parasitológico en heces, RPC) y las diferentes prevalencias de SII y Blastocystis en los lugares estudiados, podría explicar en parte los resultados discordantes en relación a establecer una relación entre SII y $B$. hominis. En Chile, tal como se mencionó previamente, en un estudio preliminar realizado por Madrid AM, y cols., se estableció una frecuencia de parasitosis intestinal en $42,5 \%$ de sujetos con SII, siendo $B$. hominis el más frecuentemente reportado ( $94 \%$ de los (asos) $)^{33}$.

\section{Entamoeba histolytica}

El complejo E. histolytica/dispar es un protozoo parásito que habita en la mucosa del colon y puede presentar principalmente dos fases o estadios durante su ciclo de vida: la forma de trofozoito, y la forma de quiste, esta última capaz de moverse y producir injuria en la mucosa intestinal del hospedero ${ }^{47}$.

Las parasitosis por E. histolytica/dispar tienen alta prevalencia en el mundo y varía según el área geográfica, siendo más frecuente en zonas tropicales y países subdesarrollados. Se reportan prevalencias que oscilan de 1 a $40 \%$ en América Central, del Sur, Asia y África y 0,2 a $10,8 \%$ en países industrializados o desarrollados. Sólo $10 \%$ de los infectados son sintomáticos, presentado disentería y dolor abdominal agudo. Menos frecuente son las manifestaciones de diarrea acuosa o deposiciones con abundante mucosidad ${ }^{48}$.

Los primeros estudios que visualizaron una asociación entre la disentería amebiana y el desarrollo de SII fueron realizados en soldados británicos al fin de la II Guerra Mundial $^{21,49}$. Posteriormente, estudios realizados en India entre los años 1984 y $1997^{50-52}$, no lograron demostrar una relación entre la presencia de síntomas de SII y la infección por E. histolytica. Recientemente, en un estudio caso-control realizado en Nicaragua, tampoco demostró diferencias en la presencia de $E$. histolytica entre pacientes con SII y controles ${ }^{46}$. Siendo las evidencias de asociación con SII obtenidas a partir la literatura científica internacional escasas, se desconoce el papel que podría jugar esta infestación en el desarrollo de SII en nuestro país.

\section{Giardia intestinalis}

La giardiasis constituye una de las parasitosis más comunes en el mundo. Giardia intestinalis (o duodenalis, o lamblia) es un protozoo que puede ser encontrado en la forma de dos estadios durante su ciclo de vida: como trofozoito, que provoca las manifestaciones clínicas de la giardiasis, y como quiste, el que transmite la infección a través de las heces, aguas y alimentos contaminados. En el duodeno los quistes se disuelven por el ambiente alcalino, para dar lugar a los trofozoitos asexuados que colonizan tanto duodeno, como yeyuno e íleon. Posteriormente vuelven a enquistarse y eliminarse en las heces ${ }^{53}$.

La prevalencia de G. intestinalis en países en desarrollo es de 20 a $30 \%$, a diferencia de países desarrollados, donde se le observa en 3 a $7 \%$ de la población ${ }^{54}$. En Chile, en un estudio no publicado, realizado por Gil, LC. y cols., presentados en las Jornadas Anuales de Parasitología 2012, Universidad de Chile, se reportó la incidencia de parasitosis intestinales en muestras de deposiciones de 2.554 pacientes asistentes al Hospital Clínico Universidad de Chile entre enero de 2010 y julio de 2011. Se observó que en jóvenes bajo 15 años, 19,3\% presentaba infección por algún parásito intestinal, y 30,15\% sobre 15 años de edad, destacando la presencia de B. hominis en 424 pacientes $(16,6 \%)$, de los cuales la mayoría son adultos $(61,7 \%)$, y $G$. intestinalis en 42 pacientes $(1,6 \%)$, de los cuales la mayoría eran niños $(69 \%)^{55}$. Otros estudios han evidenciado $G$. intestinalis en 5,5 a $12,6 \%$ en niños escolares y preescolares ${ }^{9,10}$, mostrando un descenso en su frecuencia respecto a años anteriores.

Giardia es un parásito que clásicamente provoca cuadros de diarrea y malabsorción. Sin embargo, se ha descrito la aparición tardía de diversas manifestaciones clínicas, las que incluye tanto manifestaciones intestinales como extra-intestinales (alteraciones oculares, artritis, alergias, complicaciones musculares, metabólicas y cognitivas, entre otras $)^{54}$. En relación a las manifestaciones intestinales, en un estudio prospectivo, Grazioli y cols. ${ }^{56}$, encontraron una prevalencia de $6,5 \%$ de infección por Giardia en pacientes con SII en biopsias duodenales y estudio de deposiciones. El autor concluye que la infección por Giardia podría ser una de las causas de los síntomas en pacientes con SII. Años antes, D'Anchino y cols. ${ }^{57}$, tras estudiar pacientes con giardiasis sintomática, en el grupo de pacientes que además cumplía criterios de SII, no logró la curación de sus síntomas tras el tratamiento antiparasitario, a diferencia del grupo sólo con infección por Giardia, llegando a la conclusión de que la infección por este agente podría exacerbar los síntomas de un SII pre-existente.

La persistencia de manifestaciones intestinales luego del tratamiento efectivo de erradicación del parásito trajo el concepto del SII-PI secundario a infección por Giardia. Estudios de seguimiento realizados en una cohorte de pacientes infectados y tratados durante un brote de Giardia en el 2004 en Noruega, constataron que 46,1\% de los sujetos presentaron síntomas abdominales persistentes con criterios de SII a los tres años, y 39,4\% a los seis años de seguimiento, versus un grupo control (14 y 11,6\%, 
respectivamente), con un riesgo relativo (RR) ajustado de 3,4 de padecer SII-PI asociados a Giardia ${ }^{58,59}$. En base a estos antecedentes, los autores concluyen que la infección por $G$. intestinalis podría ser un factor desencadenante de SII. Mecanismos que intentan explicar estos resultados apuntan como factor de riesgo una prolongada infección por Giardia, con demostración de una alta prevalencia de inflamación de la mucosa en biopsias duodenales post giardasis en pacientes con síntomas abdominales persistentes, compatible con un estado de inflamación crónica de bajo grado, similar a lo encontrado en biopsias intestinales de pacientes con $\mathrm{SII}^{60,61}$.

Los resultados presentados en relación al papel de los parásitos en el SII son insuficientes. Si bien existe una tendencia a una asociación positiva entre ambas entidades y en el papel de los parásitos en el SII-PI, se requieren estudios que puedan confirmar estos hallazgos en nuestro país al igual que el impacto en el manejo precoz de estas parasitosis.

\section{Conclusión}

En la fisiopatología del SII se han descrito numerosos factores, entre ellos factores psicosociales, ambientales y biológicos. Dentro de los factores biológicos se incluyen los antecedentes de infecciones, tanto bacterianas como virales y parasitarias, lo que ha permitido reconocer al subgrupo SII-PI. Se ha observado el predominio de un estado inflamatorio de bajo grado en la mucosa intestinal en pacientes con SII, cuya causa no ha sido completamente esclarecida. La presencia de esta respuesta inmune activada en la mucosa, asociada a la sintomatología visceral $y$ alteraciones en función digestiva, puede estar relacionada a una respuesta crónica de memoria que resulta de los mecanismos de defensa del hospedero frente a las infecciones gastrointestinales agudas.
En esta revisión se han presentado parte de los antecedentes que apoyan la hipótesis que la presencia de infecciones parasitarias puede ser causa de SII. El tipo de respuesta inmune activada por una parasitosis intestinal se asemeja a la observada en los pacientes con SII, en los que se destaca una aumentada activación de mastocitos en el intestino en proximidad con terminaciones nerviosas entéricas. Dentro de los agentes parasitarios asociados al SII están B. hominis, E. hystolitica y G. intestinalis. Debido a que nuestro país ha experimentado diversos cambios socioeconómicos y culturales, que repercuten en políticas de salud pública, se hace necesario extender y profundizar en el conocimiento actual de esta asociación, a través de cohortes mayores de individuos, incluyendo diversas áreas geográficas.

\section{Resumen}

El síndrome de intestino irritable (SII) es un trastorno funcional digestivo de etiología multifactorial. En su fisiopatología se describen diversos factores, tanto biológicos, como psicológicos y ambientales, que afectan el estado de activación de células inmunes en la mucosa intestinal. Entre los factores ambientales se incluye la presencia de alguna parasitosis intestinal. El síndrome de intestino irritable post-infeccioso (SII-PI) es reconocido como un subgrupo de estos trastornos, cuya aparición de los síntomas es posterior a una infección intestinal provocada por agentes microbianos. A pesar de que en Chile hay pocos estudios respecto a la relación entre SII y parasitosis intestinal, se ha descrito la existencia de una asociación positiva entre SII e infecciones por Blastocistis hominis, uno de los parásitos prevalentes en Chile. En otros países, se ha descrito además una relación entre SII, amebiasis y giardiasis. Por la alta prevalencia de parasitosis en nuestro país, existe la necesidad de ampliar los estudios para clarificar la fuerza de la asociación entre parasitosis y SII.

\section{Referencias bibliográficas}

1.- Quigley E M, Abdel-Hamid H, Barbara G, Bhatia S J, Boeckxstaens G, De Giorgio R, et al. A global perspective on irritable bowel syndrome: a consensus statement of the World Gastroenterology Organisation Summit Task Force on irritable bowel syndrome. J Clin Gastroenterol 2012; 46 (5): 356-66.

2.- Madrid-Silva A M, Defilippi-Caffri C, Landskron-Ramos G, Olguín-Herrera F, ReyesPonce A, Castro-Lara A, et al. The prevalence of irritable bowel symptoms in a population of shopping mall visitors in Santiago de Chile. Rev Gastroenterol Mex 2013; 78 (4): 203-10.

3.- Monnikes H. Quality of life in patients with irritable bowel syndrome. J Clin Gastroenterol 2011; 45 Suppl: S98-101.

4.- Canavan C, West J, Card T. Review article: the economic impact of the irritable bowel syndrome. Aliment Pharmacol Ther 2014; 40 (9): 1023-34.

5.- Tanaka Y, Kanazawa M, Fukudo S, Drossman D A. Biopsychosocial model of irritable bowel syndrome. J Neurogastroenterol Motil 2011; 17 (2): 131-9.

6.- Stark D, van Hal S, Marriott D, Ellis J,
Harkness J. Irritable bowel syndrome: a review on the role of intestinal protozoa and the importance of their detection and diagnosis. Int J Parasitol 2007; 37 (1): 11-20.

7.- $\quad$ Apt W. Epidemiología y parasitosis. In: McGraw-Hill, editor. Parasitología Humana. $1^{\circ}$ ed. Santiago, Chile 2013. p. 104-13.

8.- Vigilancia de diarreas por agentes parasitarios en menores de 5 años. Chile 2008-2012. Boletín Instituto de Salud Pública de Chile. 2012 http://www.ispch.cl/sites/default/files/ boletin_diarreas.pdf

9.- Mercado R, Castillo D, Muñoz V, Sandoval L, 
Jercic M I, Gil L C. Infecciones por protozoos y helmintos intestinales en pre-escolares y escolares de la Comuna de Colina, Santiago, Chile. Parasitol Latinoam 2003; 58: 173-6.

10.- Vidal S, Toloza L, Cancino B. Evolution of the prevalence the enteroparasitoses in Talca-Chile. Rev Chilena Infectol 2010; 27 (4): 336-40.

11.- Lee Y J, Park K S. Irritable bowel syndrome: emerging paradigm in pathophysiology. World $\mathrm{J}$ Gastroenterol 2014; 20 (10): 2456-69.

12.- Rome-Foundation. Rome III Diagnostic Criteria for Functional Gastrointestinal Disorders. http:// wwwromecriteriaorg/criteria/.2006.

13.- Longstreth G F, Thompson W G, Chey W D, Houghton L A, Mearin F, Spiller R C. Functional bowel disorders. Gastroenterology 2006; 130 (5): 1480-91.

14.- Ohman L, Tornblom H, Simren M. Crosstalk at the mucosal border: importance of the gut microenvironment in IBS. Nat Rev Gastroenterol Hepatol 2015; 12 (1): 36-49.

15.- Gazouli M, Wouters M M, Kapur-Pojskic L, Bengtson M B, Friedman E, Nikcevic G, et al. Lessons learned-resolving the enigma of genetic factors in IBS. Nat Rev Gastroenterol Hepatol 2016; 13 (2): 77-87.

16.- Rajilic-Stojanovic M, Jonkers D M, Salonen A, Hanevik K, Raes J, Jalanka J, et al. Intestinal microbiota and diet in IBS: causes, consequences, or epiphenomena? Am J Gastroenterol 2015; 110 (2): 278-87.

17.- Vicario M, González-Castro A M, Martínez C, Lobo B, Pigrau M, Guilarte M, et al. Increased humoral immunity in the jejunum of diarrhoea-predominant irritable bowel syndrome associated with clinical manifestations. Gut 2015; 64 (9): 1379-88.

18.- Moloney R D, Johnson A C, O’Mahony S M, Dinan T G, Greenwood-Van Meerveld B, Cryan J F. Stress and the microbiota-gut-brain axis in visceral pain: relevance to irritable bowel syndrome. CNS Neurosci Ther 2016; 22 (2): 102-17.

19.- Thabane M, Marshall J K. Post-infectious irritable bowel syndrome. World J Gastroenterol 2009; 15 (29): 3591-6.

20.- Grover M, Camilleri M, Smith K, Linden D R, Farrugia G. On the fiftie ${ }^{\text {th }}$ anniversary. Postinfectious irritable bowel syndrome: mechanisms related to pathogens. Neurogastroenterol Motil 2014; 26 (2): 156-67.

21.- Chaudhary N A, Truelove S C. The irritable colon syndrome. A study of the clinical features, predisposing causes, and prognosis in 130 cases. Q J Med 1962; 31: 307-22.

22.- Motomura Y, Khan W I, El-Sharkawy R T, Verma-Gandhu M, Grencis R K, Collins S M. Mechanisms underlying gut dysfunction in a murine model of chronic parasitic infection. Am J Physiol Gastrointest Liver Physiol 2010; 299 (6): G1354-60.

23.- Beatty J K, Bhargava A, Buret A G. Post- infectious irritable bowel syndrome: mechanistic insights into chronic disturbances following enteric infection. World J Gastroenterol 2014; 20 (14): 3976-85.

24.- Ohman L, Isaksson S, Lindmark A C, Posserud I, Stotzer P O, Strid H, et al. T-cell activation in patients with irritable bowel syndrome. Am J Gastroenterol 2009; 104 (5): 1205-12.

25.- Dinan T G, Quigley E M, Ahmed S M, Scully P, O’Brien S, O'Mahony L, et al. Hypothalamicpituitary-gut axis dysregulation in irritable bowel syndrome: plasma cytokines as a potential biomarker? Gastroenterology 2006; 130 (2): 304-11.

26.- Liebregts T, Adam B, Bredack C, Roth A, Heinzel S, Lester $\mathrm{S}$, et al. Immune activation in patients with irritable bowel syndrome. Gastroenterology 2007; 132 (3): 913-20.

27.- Dunlop S P, Jenkins D, Spiller R C. Distinctive clinical, psychological, and histological features of postinfective irritable bowel syndrome. Am J Gastroenterol 2003; 98 (7): 1578-83.

28.- Bercik P, Wang L, Verdu E F, Mao Y K, Blennerhassett P, Khan W I, et al. Visceral hyperalgesia and intestinal dysmotility in a mouse model of postinfective gut dysfunction. Gastroenterology 2004; 127 (1): 179-87.

29.- Barbara G, Stanghellini V, De Giorgio R, Cremon C, Cottrell G S, Santini D, et al. Activated mast cells in proximity to colonic nerves correlate with abdominal pain in irritable bowel syndrome. Gastroenterology 2004; 126 (3): 693-702.

30.- Lee Y A, Nam Y H, Min A, Kim K A, Nozaki T, Saito-Nakano Y, et al. Entamoeba histolytica-secreted cysteine proteases induce IL-8 production in human mast cells via a PAR2-independent mechanism. Parasite 2014; 21: 1 .

31.- Hardin J A, Buret A G, Olson M E, Kimm M H, Gall D G. Mast cell hyperplasia and increased macromolecular uptake in an animal model of giardiasis. J Parasitol 1997; 83 (5): 908-12.

32.- Spiller R, Garsed K. Postinfectious irritable bowel syndrome. Gastroenterology 2009; 136 (6): 1979-88.

33.- Madrid A M. Gil L C, Defilippi C L, Lanskron G, Castro A. ¿Debemos estudiar infección intestinal por parásitos en pacientes con Síndrome de Intestino Irritable? Rev Gastroenterología Latinoamericana 2011; 22 (1): 44.

34.- Apt W. Blastocistiasis. In: McGraw-Hill, editor. Parasitología Humana. $1^{\circ}$ ed. Santiago, Chile 2013. p. 152-4.

35.- Wawrzyniak I, Poirier P, Viscogliosi E, Dionigia M, Texier C, Delbac F, et al. Blastocystis, an unrecognized parasite: an overview of pathogenesis and diagnosis. Ther Adv Infect Dis 2013; 1 (5): 167-78.

36.- Poirier P, Wawrzyniak I, Vivares C P, Delbac F, El Alaoui H. New insights into Blastocystis spp: a potential link with irritable bowel syndrome. PLoS Pathog 2012; 8 (3): e1002545.

37.- Giacometti A, Cirioni O, Fiorentini A, Fortuna $\mathrm{M}$, Scalise G. Irritable bowel syndrome in patients with Blastocystis hominis infection. Eur J Clin Microbiol Infect Dis 1999; 18 (6): 436-9.

38.- Jiménez-González D E, Martínez-Flores W A, Reyes-Gordillo J, Ramírez-Miranda M E, Arroyo-Escalante S, Romero-Valdovinos M, et al. Blastocystis infection is associated with irritable bowel syndrome in a Mexican patient population. Parasitol Res 2012; 110 (3): 126975 .

39.- Yakoob J, Jafri W, Beg M A, Abbas Z, Naz S, Islam $\mathrm{M}$, et al. Irritable bowel syndrome: is it associated with genotypes of Blastocystis hominis. Parasitol Res 2010; 106 (5): 1033-8.

40.- Dogruman-Al F, Simsek Z, Boorom K, Ekici E, Sahin M, Tuncer C, et al. Comparison of methods for detection of Blastocystis infection in routinely submitted stool samples, and also in IBS/IBD patients in Ankara, Turkey. PLoS One 2010; 5 (11): e15484.

41.- Yakoob J, Jafri W, Jafri N, Khan R, Islam M, Beg M A, et al. Irritable bowel syndrome: in search of an etiology: role of Blastocystis hominis. Am J Trop Med Hyg 2004; 70 (4): 383-5.

42.- Yakoob J, Jafri W, Beg M A, Abbas Z, Naz $\mathrm{S}$, Islam M, et al. Blastocystis hominis and Dientamoeba fragilis in patients fulfilling irritable bowel syndrome criteria. Parasitol Res 2010; 107 (3): 679-84

43.- Tungtrongchitr A, Manatsathit S, Kositchaiwat C, Ongrotchanakun J, Munkong N, Chinabutr P, et al. Blastocystis hominis infection in irritable bowel syndrome patients. Southeast Asian J Trop Med Public Health 2004; 35 (3): 705-10.

44.- Ramírez-Miranda M E, Hernández-Castellanos R, López-Escamilla E, Moncada D, RodríguezMagallan A, Pagaza-Melero C, et al. Parasites in Mexican patients with irritable bowel syndrome: a case-control study. Parasit Vectors 2010; 3: 96.

45.- Surangsrirat $\mathrm{S}$, Thamrongwittawatpong $\mathrm{L}$, Piyaniran W, Naaglor T, Khoprasert C, Taamasri P, et al. Assessment of the association between Blastocystis infection and irritable bowel syndrome. J Med Assoc Thai 2010; 93 Suppl 6: S119-24.

46.- Morgan D R, Benshoff M, Cáceres M, Becker-Dreps S, Cortés L, Martin C F, et al. Irritable bowel syndrome and gastrointestinal parasite infection in a developing nation environment. Gastroenterol Res Pract 2012; 2012: 343812. doi: 10.1155/2012/343812. Epub 2012 Feb 19

47.- Thibeaux R, Weber C, Hon C C, Dillies M A, Ave P, Coppee J Y, et al. Identification of the virulence landscape essential for Entamoeba histolytica invasion of the human colon. PLoS Pathog 2013; 9 (12): e1003824. 
48.- Chacin-Bonilla L. An update on amebiasis. Rev Med Chile 2013; 141 (5): 609-15.

49.- Stewart G T. Post-dysenteric colitis. Br Med J 1950; 1 (4650): 405-9.

50.- Nanda R, Baveja U, Anand B S. Entamoeba histolytica cyst passers: clinical features and outcome in untreated subjects. Lancet 1984; 2 (8398): 301-3.

51.- Anand A C, Reddy P S, Saiprasad G S, Kher S K. Does non-dysenteric intestinal amoebiasis exist? Lancet 1997; 349 (9045): 89-92.

52.- Sinha P, Ghoshal U C, Choudhuri G, Naik S, Ayyagari A, Naik S R. Does Entamoeba histolytica cause irritable bowel syndrome? Indian J Gastroenterol 1997; 16 (4): 130-3.

53.- Apt W. Giardiasis. In: McGraw-Hill, editor. Parasitología Humana. $1^{\circ}$ ed. Santiago, Chile 2013. p. $145-51$.

54.- Halliez M C, Buret A G. Extra-intestinal and long term consequences of Giardia duodenalis infections. World J Gastroenterol 2013; 19 (47): 8974-85.

55.- Gil L C, Hernández E, Madrid A M, Hurtado C, Castro A, Alcalde M. Parasitosis Intestinales ¿Una realidad vigente en nuestro medio? In: (SOCHIPA) SCdP, editor. XIV Jornadas Anuales de Parasitología; Santiago, Chile 2012. p. 44.

56.- Grazioli B, Matera G, Laratta C, Schipani G, Guarnieri G, Spiniello E, et al. Giardia lamblia infection in patients with irritable bowel syndrome and dyspepsia: a prospective study. World J Gastroenterol 2006; 12 (12): 1941-4.

57.- D'Anchino M, Orlando D, De Feudis L. Giardia lamblia infections become clinically evident by eliciting symptoms of irritable bowel syndrome. J Infect 2002; 45 (3): 169-72.

58.- Wensaas K A, Langeland N, Hanevik K, Morch K, Eide G E, Rortveit G. Irritable bowel syndrome and chronic fatigue 3 years after acute giardiasis: historic cohort study. Gut 2012; 61 (2): 214-9.

59.- Hanevik K, Wensaas K A, Rortveit G, Eide G E, Morch K, Langeland N. Irritable bowel syndrome and chronic fatigue 6 years after giardia infection: a controlled prospective cohort study. Clin Infect Dis 2014; 59 (10): 1394-400.

60.- Hanevik K, Hausken T, Morken M H, Strand E A, Morch K, Coll P, et al. Persisting symptoms and duodenal inflammation related to Giardia duodenalis infection. J Infect 2007; 55 (6): 524-30.

61.- Morch K, Hanevik K, Rortveit G, Wensaas K A, Eide G E, Hausken T, et al. Severity of Giardia infection associated with post-infectious fatigue and abdominal symptoms two years after. BMC Infect Dis 2009; 9: 206. 